\title{
Tsafon
}

Revue d'études juives du Nord

$81 \mid 2021$

Des synagogues à travers les âges Lieux de prières, lieux d'études et autres fonctions

\section{Fanny Kleiman (1929-2021) La petite fille cachée sous la table}

Danielle Delmaire

\section{(2) OpenEdition}

Journals

Édition électronique

URL : https://journals.openedition.org/tsafon/3965

DOI : $10.4000 /$ tsafon.3965

ISSN : 2609-6420

Éditeur

Association Jean-Marie Delmaire

Édition imprimée

Date de publication : 1 juillet 2021

Pagination : 145-150

ISSN : $1149-6630$

Référence électronique

Danielle Delmaire, «Fanny Kleiman (1929-2021) La petite fille cachée sous la table», Tsafon [En ligne], 81 | 2021, mis en ligne le 01 juillet 2021, consulté le 15 septembre 2021. URL : http:// journals.openedition.org/tsafon/3965; DOI : https://doi.org/10.4000/tsafon.3965 


\section{Hommage}

\section{Fanny Kleiman (1929-2021) \\ La petite fille cachée sous la table}

Danielle Delmaire*

Au début de ce mois d'avril 2021, Fanny Kleiman, née Dawidowicz, s'en est allée et nous souhaitons rendre hommage à une fidèle lectrice et abonnée de Tsafon. Dès les premiers numéros, elle a accompagné et soutenu l'aventure de la revue ; depuis, elle n'a jamais rompu sa fidélité. C'est aussi de la petite fille cachée sous la table, pour échapper à l'arrestation, dont nous nous souvenons.

Fanny était née dans une famille immigrée de Pologne, résidant à Béthune à la fin des années 1930. Joseph Dawidowicz, né en 1886, était originaire de la région de Lodz; après avoir mené une vie d'errance à travers l'Europe, il revint dans son village pour épouser Hana Gliksman. Le couple quitta la Pologne en 1919 pour s'installer dans « le pays des droits de l'Homme », la France, et démarrer une nouvelle existence à Douai puis à Béthune en 1936 où il «investit dans un magasin de vêtements pour dames $»^{1}$. Le couple eut quatre enfants : Jean (né en 1921), Jenny (née en 1926), Fanny (née en 1929) et Simon (né en 1930). La famille n'était pas très pratiquante mais respectait les principales fêtes juives. Le souci des parents était de s'intégrer dans la société française et

\footnotetext{
* Laboratoire ALITHILA, université de Lille.

${ }^{1}$ Ces renseignements biographiques et les suivants proviennent du témoignage de Simon Dawidowicz, jeune frère de Fanny : Clandestins sous l'Occupation, livre édité et diffusé à compte d'auteur, 2008, p. 3-49.
} 
la fréquentation, par les enfants, de l'école de la République devait largement y contribuer. Le cercle familial s'était élargi avec la présence d'un autre couple : les Gliksman, à Douai et la famille d'un frère de Joseph à Avion.

Jusqu'à l'invasion de la Belgique par les Allemands et l'occupation de la partie nord de la France, la vie s'écoule paisiblement pour ces familles immigrées qui ont fui l'antisémitisme latent et permanent dans la Pologne d'avant-guerre. Puis c'est l'exode vers le sud, suivi rapidement d'un retour à Béthune où le magasin a été pillé. Mais la vie reprend tant bien que mal et: " nous les enfants, nous reprenons l'école $»^{2}$. Pas pour longtemps car les directives qui proviennent de Bruxelles puisque le Nord et le Pas-de-Calais sont rattachés, dès les premiers jours de l'occupation, au gouvernement militaire de Bruxelles, prévoient de rendre le littoral de la Côte d'Opale « sans juifs » et de procéder à l'arrestation et l'internement des juifs dans un centre de regroupement pour étrangers à Troyes ${ }^{3}$. Le littoral s'étend loin dans les terres puisque Béthune en fait partie. La famille Dawidowicz est donc menacée. C'est sans compter sur l'énergie des parents qui, prévenus du danger, décident de partir immédiatement, une seconde fois, se réfugiant avec leur fille Jenny chez des amis, les Caine qui résident à Nœux-les-mines près de Béthune, et confiant les deux petits derniers au couple Delestrez, concierges de la mairie de Béthune qui jouxte le magasin, tandis que l'aîné Jean part de son côté. C'est ainsi que le 16 décembre 1940, alors qu'ils viennent arrêter une famille juive, les Allemands trouvent le domicile des Dawidowicz vide.

Commence alors pour Fanny et son petit frère Simon une vie relativement douce même s'ils sont séparés de leurs parents car les Delestrez se montrent très accueillants. Toutefois, ils ne peuvent pas sortir ni se rendre à l'école. Par deux fois, ils échappent à une perquisition. C'est lors de l'une d'elles que les enfants se dissimulent sous une table dont la toile cirée descend jusqu'au sol tandis que les Allemands sont invités, par $\mathrm{M}^{\mathrm{me}}$ Delestrez, à déguster un café à cette même table !

Fanny et son frère ne peuvent rester dans cette situation instable et dangereuse aussi ce refuge ne dure que deux mois. Les parents qui ont quitté, avec leur fille Jenny, la région minière pour Paris, décident de

\footnotetext{
${ }^{2}$ Ibid., p. 10. Le frère aîné, Jean, a aussi écrit ses souvenirs : « Un couple dans la guerre. Témoignage de Jean et Charlotte Dawidowicz », Tsafon, revue d'études juives du Nord, $n^{\circ} 47$, printemps - été 2004, p. 41-61.

${ }^{3}$ Lire Rudy Rigaut, « Les particularités la zone côtière dans la persécution des Juifs dans le Nord et le Pas-de-Calais occupés (1940-1944) », Tsafon revue d'études juives du Nord, n 79 , printemps - été 2020, p. 39-74.
} 
reprendre les enfants. C'est l'aîné Jean qui, aidé d'amis déjà impliqués dans des actes de résistance, parvient à faire sortir ses frère et sœur de la mairie, dans des sacs de pommes de terre, pour les charger dans une camionnette qui les évacue hors de la ville. Puis ils gagnent Paris où se trouvent les parents qui décident, une fois encore, de se réfugier en zone libre, à Pau. Ils y louent un appartement et les enfants retrouvent l'école.

Toutefois, ces moments de relative sérénité ne durent pas. Après l'invasion de la zone sud, en novembre 1942, de nouveau la famille se sent en danger et les parents tranchent : il faut disperser les enfants, Fanny et Simon sont confiés à un couple de protestants, les Cadier qui vivent au lieu-dit reculé de Latapy, au sud de Pau. Ils sont accueillis sans condition et partagent une vie de famille heureuse, au bon air de la montagne. Jenny est cachée dans une autre famille et Jean entame un parcours de résistant qui le mène en Isère ${ }^{4}$. Fanny et Simon restent chez les Cadier de la fin de 1942 jusqu'au début de 1944 : une menace de dénonciation oblige les parents à reprendre les enfants auprès d'eux à Tullins-Fures, non loin de Grenoble, puis à Saint-Quentin en Isère. Là encore, la famille échappe à plusieurs arrestations et chacun doit adopter une fausse identité.

À la Libération, toute la famille peut enfin se réunir et retourner à Béthune pour constater que le magasin a été "aryanisé» et liquidé. Toutefois, le préjudice est relatif car, parmi les acheteurs, se trouvent des amis, décidés à rendre leurs acquisitions au propriétaire légitime, dès son retour. Ce qui a été fait.

Fanny et sa famille ont entamé des démarches auprès de Yad Vashem pour que soit accordé aux familles Delestrez, Caine et Cadier, le titre de Justes parmi les Nations. Le 4 septembre 1991, les familles Delestrez et Cadier étaient reconnues Justes tandis que la famille Caine l'était le 19 février 1992. La cérémonie du 8 mars 1992 qui honora les Delestrez s'est déroulée à la mairie de Béthune, au-dessus de laquelle se situait l'appartement où Fanny et Simon furent cachés.

Retenons, dans ce récit de sauvetage d'une famille juive, que les décisions de se soustraire à la déportation appartiennent aux parents : Joseph et Hanna. Tous deux sont bien décidés à résister et à refuser la persécution. Certes, des amis les préviennent des arrestations imminentes comme celle du 16 décembre 1940 à Béthune, et, certes, d'autres amis les accueillent : les Caine, les Cadier mais ce sont eux qui les sollicitent. Cette famille résiste, sans armes, et finit par survivre à la guerre. Leur parcours constitue la preuve que tous les juifs ne sont pas partis à l'abattoir sans

\footnotetext{
${ }^{4}$ Lire son témoignage, voir note 2 .
} 
s'opposer. C'est ce que constatent les historiens Jacques Semelin et Monique Heddebaut, entre autres ${ }^{5}$.

La famille Gliksman, de Douai dont cinq enfants, et la famille Dawidowicz, d'Avion près de Lens, dont trois enfants, furent raflés le 11 septembre 1942 pour être internés à la caserne Dossin à Malines près d'Anvers et être déportés à Auschwitz, sans retour, le 15 septembre 1942.

Plusieurs petits-enfants de Joseph et Hanna ont eu à cœur de faire connaître l'histoire du sauvetage de leur famille. Pour son excellent travail sur la communauté juive de Lens pendant la guerre, Claire Zalc a bénéficié de leurs récits. Le prologue débute par « Joseph et les siens » pour placer l'étude « aux origines de l'histoire ${ }^{6}$. Il s'agit bien de Joseph Dawidowicz.

Le fils de Fanny, Thierry Kleiman, m'a accordé de très nombreuses heures pour m'aider à dresser la liste des personnes raflées le 11 septembre 1942, parue dans un des premiers numéros de Tsafon, celui-là même où Fanny raconte sa cachette sous la table des Delestrez ${ }^{7}$. C'était pour lui primordial de sacrifier son temps à la mémoire des déportés parmi lesquels se trouvaient des membres de sa famille.

Paulette Touzard-Dawidowicz, fille aînée de Jean et née dans les derniers mois de la guerre, s'investit actuellement dans l'Association pour la Mémoire des Enfants Juifs Déportés (AMEJD) qu'elle préside pour le Nord et elle se donne pour tâche l'apposition de plaques commémoratives dans les établissements scolaires où ont étudié des enfants déportés.

Jenny épousa Nathan Alpern qui fut raflé, avec ses parents, le 11 septembre 1942 et qui revint de déportation malgré ses passages à Auschwitz et à Dora (seul juif nordiste ayant été interné dans ce camp) ${ }^{8}$.

\footnotetext{
${ }^{5}$ C'est la thèse développée par Jacques Semelin qui explique que les juifs ont pris leur sort en mains pour réussir leur sauvetage. Lire : La survie des juifs de France. 1940-1944, Paris, CNRS éditions, 2013. Dans son sillage, Monique Heddebaut adopte la même position concernant les rescapés de la rafle du 11 setptembre1942 dans le Nord et le Pasde-Calais. Lire: "Sans armes face à la rafle du 11 septembre 1942, dans la 'Zone rattachée' à Bruxelles », Tsafon, revue d'études juives du Nord, $\mathrm{n}^{\circ}$ 70, automne $2015-$ hiver 2016, p. 119-168. Le titre reprend volontairement celui d'un autre ouvrage de Jacques Semelin : Sans armes face à Hitler : la résistance civile en Europe, 1939-1943, Paris, Payot, 1989.

${ }^{6}$ Nicolas Mariot et Claire Zalc, Face à la persécution, 991 juifs dans la guerre, Paris, Odile Jacob, 2010, p. 11.

7 «Liste des déportés du convoi $X »$ dressée par Danielle Delmaire et Thierry Kleiman, Tsafon, revue d'études juives du Nord, n 9-10, été-automne 1992, p. 38-66. La liste fut réalisée à partir d'un document d'archives qui, dans les années 1980, se trouvait au Ministère de la Santé Publique et de la Famille à Bruxelles.

${ }^{8}$ Lire sa notice dans Laurent Thiery (sous la dir. de), Le Livre des 9000 déportés de France à Mittelbau-Dora, Paris, éd. du Cherche Midi, 2020.
} 
Après la guerre, il enseigna l'allemand dans un lycée de la région et organisa des échanges scolaires entre jeunes Allemands et jeunes Français. La ville de Béthune a honoré sa mémoire en nommant une rue à son nom : Philippe Alpern (prénom qu'il adopta après la guerre), en 1992, et une salle de la mairie en 2009, rebaptisée après quelques années durant lesquelles la photo qui orne la salle avait disparu? ${ }^{9}$.

Fanny avait partagé sa vie avec Henri Tajchner, déporté à Auschwitz et revenu malgré un internement de trois ans ${ }^{10}$.

En souvenir, nous reproduisons une partie du témoignage de Fanny, cachée sous la table des Delestrez, paru dans le n 9-10 de Tsafon (été automne 1992, p. 8-10) :

\begin{abstract}
Nous sommes restés plusieurs mois chez M. et $\mathrm{M}^{\mathrm{me}}$ Delestrez. Ils étaient vraiment gentils avec nous $[\ldots]$

Et puis un jour quelqu'un a su que les enfants Dawidowicz étaient cachés chez les concierges de la mairie, à deux pas de la Kommandantur. Peu de temps après, il y eut une descente d'Allemands chez $\mathrm{M}^{\mathrm{me}}$ Delestrez. Ils devaient monter quatre étages et, avec leurs bottes, ils faisaient du bruit si bien qu'on les entendit monter. $\mathrm{M}^{\mathrm{me}}$ Delestrez eut juste le temps de nous cacher sous la table, Simon et moi, en tirant sur la toile cirée. L'appartement n'avait pas d'autre issue que la porte d'entrée, impossible de fuir. Les Allemands entrèrent donc et réclamèrent les enfants juifs. Avec un aplomb extraordinaire, $\mathrm{M}^{\mathrm{me}}$ Delestrez les invita à fouiller toute la maison y compris les armoires. Ils jetèrent un regard rapide dans la pièce qui n'avait qu'une porte, alors elle ajouta toujours avec le même aplomb : «Vous prendrez bien une tasse de café ». Ils s'assirent là, tous les deux ou les trois, je ne me souviens plus, autour de la table, et Simon et moi nous nous blottissions comme deux petits oiseaux, serrés l'un contre l'autre. Il fallait surtout ne faire aucun bruit, ni tousser, ni éternuer, ni même respirer. Dans cette cachette précaire, le temps nous paraissait terriblement long. Enfin ils burent leur café. Sous la table, je me vis dans une glace accrochée face à moi mais les Allemands ne pouvaient pas m'apercevoir. Et je pensais que s'ils nous voyaient, ils nous prendraient. J'étais paralysée par la peur ce jour-là et d'ailleurs si je suis si nerveuse aujourd'hui, ce n'est pas sans raison. Et puis les Allemands partirent.

Les journées s'écoulèrent douces mais j'étais triste car je n'avais plus mes parents. Les Allemands effectuèrent une deuxième descente, ce jour-là $\mathrm{M}^{\mathrm{me}}$ Delestrez nous cacha derrière une grande armoire. Elle avait placé une caisse de bière sur laquelle nous étions montés si bien que si les Allemands regardaient sous l'armoire, ils ne pouvaient même pas distinguer nos pieds.
\end{abstract}

\footnotetext{
${ }^{9}$ Lire Danielle Delmaire, «La commémoration de la persécution antijuive durant la Seconde Guerre mondiale. Les plaques et les stèles dans le Nord/Pas-de-Calais », Tsafon, revue d'études juives $d u$ Nord, $\mathrm{n}^{\circ}$ 66, automne 2013 - hiver 2014, p. 109-130.

${ }^{10}$ Le journaliste Bruno Vouters a recueilli et a publié le témoignage d'Henri Tajchner, Trois ans dans l'enfer d'Auschwitz. Parole de survivant, 1995, $2^{\mathrm{e}}$ édition, Lille, éd. La Voix du Nord, 2012.
} 


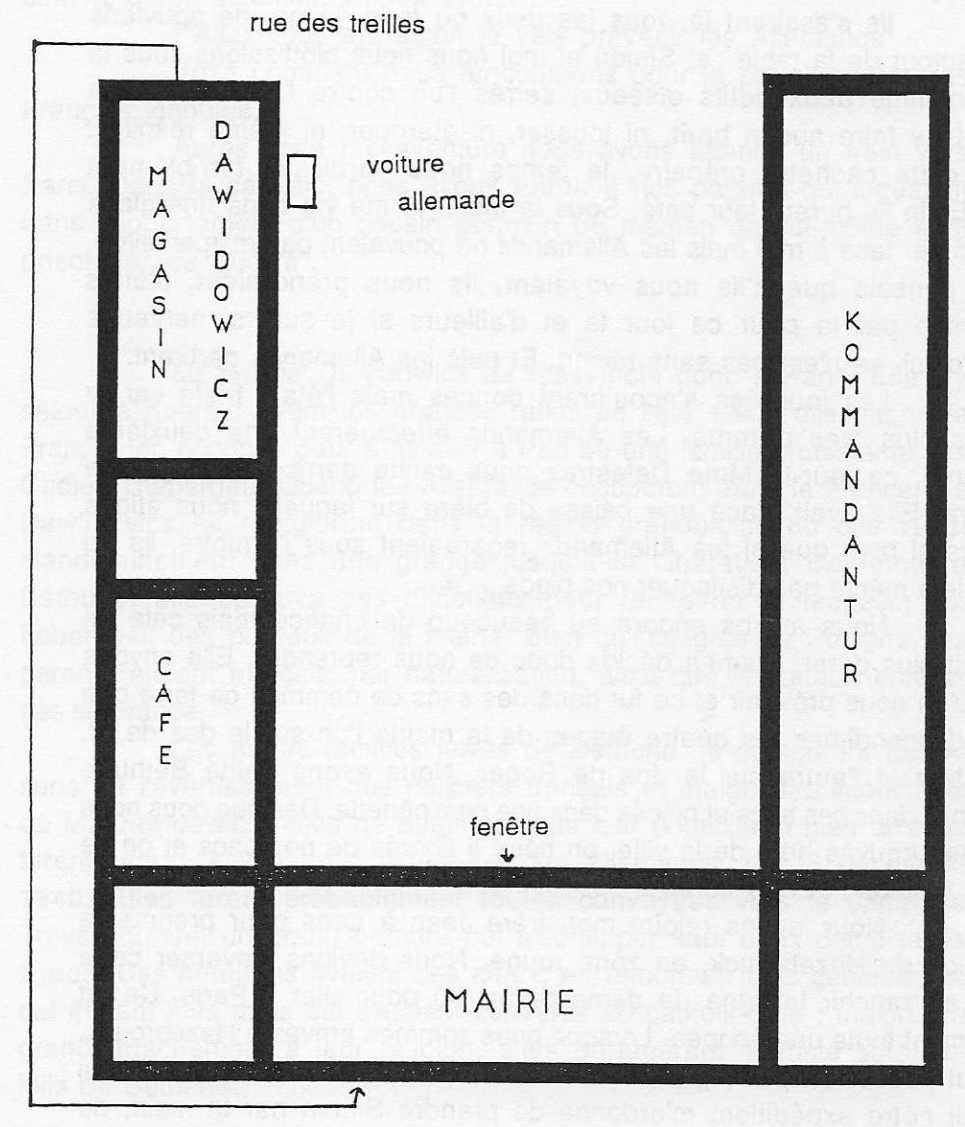

Plan paru dans le n 9-10 de Tsafon, à partir du témoignage de Fanny.

La flèche indique le trajet suivi par les enfants pour rejoindre le domicile des Delestrez.

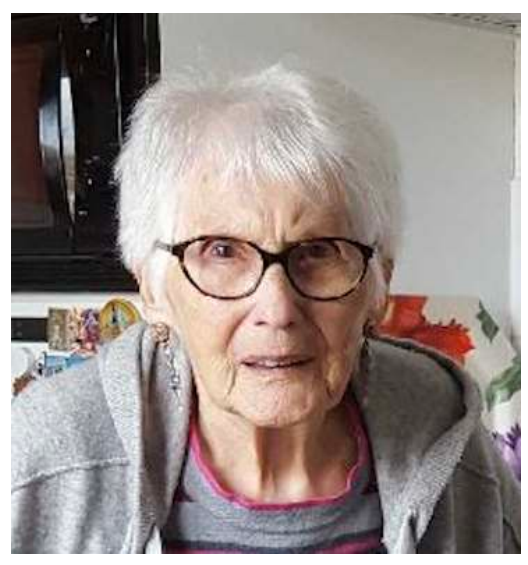
Fanny Kleiman peu d'années
avant son décès.

(archives de la famille Dawidowicz) 